\title{
Observation of Polarization Singularities at the Nanoscale
}

\author{
M. Burresi, ${ }^{1, *}$ R. J. P. Engelen, ${ }^{1}$ A. Opheij,${ }^{1}$ D. van Oosten, ${ }^{1}$ D. Mori, ${ }^{2}$ T. Baba, ${ }^{2}$ and L. Kuipers ${ }^{1}$ \\ ${ }^{1}$ Center for Nanophotonics, FOM Institute for Atomic and Molecular Physics (AMOLF), \\ Kruislaan 407, 1098 SJ Amsterdam, The Netherlands \\ ${ }^{2}$ Department of Electrical and Computer Engineering, Yokohama National University, \\ 79-5 Tokiwadai, Hodogayaku, Yokohama 240-8501, Japan
}

(Received 30 August 2008; revised manuscript received 31 October 2008; published 23 January 2009)

\begin{abstract}
With a phase-sensitive near-field microscope we measure independently the two in-plane electric field components of light propagating through a $2 \mathrm{D}$ photonic crystal waveguide and the phase difference between them. Consequently, we are able to reconstruct the electric vector field distribution with subwavelength resolution. In the complex field distribution we observe both time-dependent and timeindependent polarization singularities and determine the topology of the surrounding electric field.
\end{abstract}

DOI: 10.1103/PhysRevLett.102.033902

A wave singularity can be defined as a position in space where a property of a wave is not defined. Such singularities can be found in many types of waves and they can have a large impact on common life. For instance, they play an important role in heart fibrillation [1] and in the distribution of tides [2]. Moreover, wave singularities are studied in many different fields of physics, such as high energy physics [3], Bose-Einstein condensation [4-6], superconductivity [7], and superfluidity [8]. In optics, wave singularities have been studied since the 1830s (for an overview, see for instance Berry [9]) and have been observed in the interference patterns of the diffracted light of our natural surroundings. The simplest class of wave singularity is the so-called wave dislocation [10] (vortex, phase singularity, edge dislocation, etc.), which is a scalar field singularity that occurs where the amplitude of a complex valued field is zero and hence its phase is undefined or "singular" [11-13]. Another more subtle class is formed by the so-called polarization singularity ( $C$ points, $L$ lines, and disclinations), which occurs in vector fields at positions where one of the parameters that characterize the polarization ellipse (handedness, eccentricity, or orientation) is singular [14-16]. In the past few years polarization singularities have been investigated in optics in the context of tightly focused beams [17], crystal optics [18], skylights [19], and speckle fields $[20,21]$. The polarization state of light is of crucial importance in quantum optics. Control of the local polarization state with a nanophotonic structure could therefore be exploited to manipulate a Bose-Einstein condensate trapped close to a surface [22], an atom chip [23], or a photonic crystal [24]. In addition, a spatial dependence of the polarization state will have important consequences for the use of single emitters in photonic nanostructures [25] for quantum information processing. Near-field microscopy has already proven to be a powerful tool in studies of the polarization distribution close to photonic nanostructures [26-28].

In this work, we observe the presence of polarization singularities at the nanoscale in the fields above a $2 \mathrm{D}$
PACS numbers: 42.25.Bs, 42.25.Fx, 42.70.Qs, 42.90.+m

photonic crystal waveguide. We succeeded in the separation of the two in-plane field components above the waveguide with high selectivity. Exploiting the measured phase difference between the components, we reconstruct the distribution of the in-plane electric vector field with subwavelength resolution. We are able to observe several types of polarization singularities and the topology of the surrounding field at the nanoscale.

The setup is schematically shown in Fig. 1. The inset in the upper-left corner shows the photonic crystal waveguide used in this work. The sample consists of a silicon membrane with a $200 \mathrm{~nm}$ thickness in which a periodic triangular arrangement of holes (lattice constant $a=450 \mathrm{~nm}$ ) has been etched. The waveguide is formed by a single

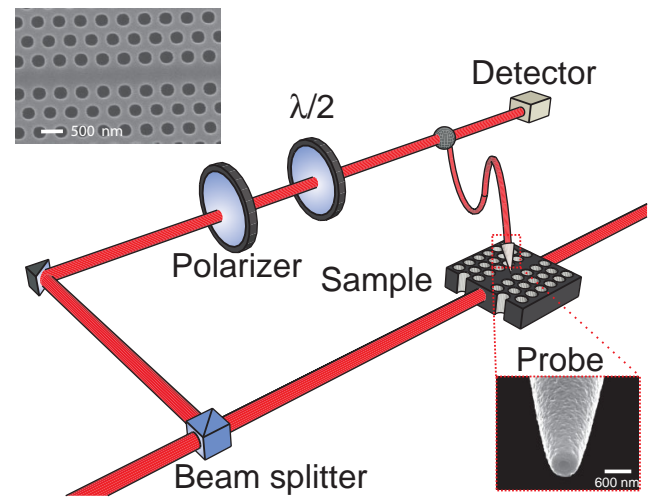

FIG. 1 (color). Schematic representation of the experimental setup. Light is coupled into a $2 \mathrm{D}$ photonic crystal waveguide. The near-field probe is scanned above the sample and collects light that is interferometrically mixed with a reference beam and detected using a heterodyne scheme. The polarization state in the reference branch is controlled using wave plates. Left inset: Scanning electron micrograph of the silicon membrane photonic crystal waveguide under investigation (lattice constant $a=450 \mathrm{~nm}$ and hole diameter $d=250 \mathrm{~nm}$ ). Right inset: Scanning electron micrograph of the aluminum-coated near-field probe with aperture of $\approx 200 \mathrm{~nm}$. 
missing row of holes. The light source used to investigate the sample is a diode laser tuned to a vacuum wavelength of $1463 \mathrm{~nm}$. The linearly polarized light is coupled into the waveguide by focusing it on the entrance facet. The field above the sample is picked up by the subwavelength aperture of an aluminum-coated near-field probe [29] (lower-right inset of Fig. 1) that is kept $20 \mathrm{~nm}$ above the sample using shear force feedback. The light collected by the probe is interferometrically mixed with a reference signal and subsequently recorded using heterodyne detection [30]. Raster scanning the near-field probe above the 2D photonic structure, we recover the phase and the amplitude pattern of the light field in the waveguide with subwavelength resolution. This allows us to reconstruct the complex electric field as $E(x, y)=A(x, y) e^{i \phi(x, y)}$, where $A$ and $\phi$ are the position-dependent amplitude and phase, respectively. Moreover, when two orthogonal polarizations are present in the probe fiber, we can select either one by choosing the appropriate polarization for the reference branch. As a result, our near-field setup is now sensitive to the polarization state of the near-field.

Figures 2(a) and 2(b) show the amplitude pattern recorded for two orthogonal polarizations of the reference signal. The amplitude is normalized to the maximum value and the scanned area is $4 a \times 5 a$. The waveguide is centered around $x=0$. Using Fourier analysis, we observe the presence of both forward and backward propagating Bloch modes in the photonic crystal waveguide [31]. We attribute the backward propagating mode to light that is reflected at the end facet of the waveguide. When we select only the

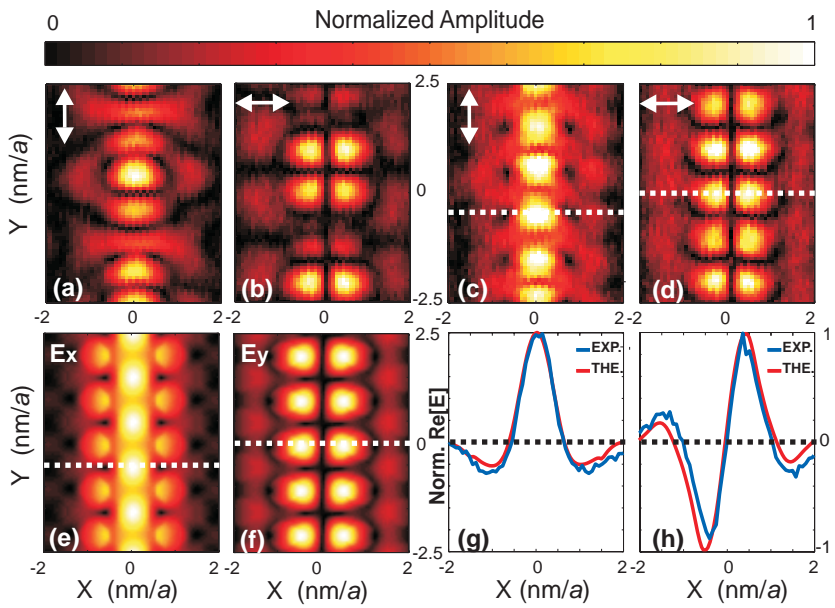

FIG. 2 (color). (a),(b) Detected amplitude pattern for two orthogonal polarizations (indicated by white arrows) in the reference branch. (c),(d) Amplitude pattern of the forward propagating mode, obtained after Fourier analysis of (a) and (b). (e), (f) Theoretical amplitude pattern of $E_{x}$ and $E_{y} 20 \mathrm{~nm}$ above the surface for the forward propagating mode. For all the images the depicted area is $4 a \times 5 a$ (the center of the waveguide is around $x=0)$ and the amplitude is normalized. (g),(h) Crosscuts of the experimentally (blue) and theoretically (red) obtained real part of the complex field along the dashed lines of (c), (d), (e), and (f), respectively. forward propagating mode by applying a Fourier filter to the experimental data [31], we obtain the amplitude distribution shown in Figs. 2(c) and 2(d). Figures 2(e) and 2(f) show the amplitude of the $E_{x}$ and $E_{y}$ components, i.e., the in-plane components, of the electric field, respectively, of the forward propagating mode, as obtained using 3D finitedifference time-domain calculations. Figures $2(\mathrm{~g})$ and $2(\mathrm{~h})$ show the line traces of the real part of the complex signals along the dashed lines of Figs. 2(c)-2(f). Blue corresponds to the measurements and red to the calculations. Whereas in the first image the line traces are symmetric, in the second the line traces have an antisymmetric pattern, with a zero crossing in the center of the waveguide. We find an excellent agreement between the measured and the calculated patterns. Thus, by utilizing a highly cylindrical symmetric aperture probe, we have succeeded in measuring the field distribution of the in-plane electric field components by using two suitably chosen orthogonal polarizations in the reference branch.

Exploiting the amplitude and the phase relation between the electric field components, we reconstruct the in-plane vector field of the electric field in a single unit cell of the photonic crystal waveguide. Figures 3(a) and 3(b) show the experimentally and theoretically obtained vector plot of the electric field. The depicted area is $2 a \times 1 a$ and the waveguide is centered around $x=0$. The contour lines indicate the measured and calculated electric field magnitude. The out-of-plane component is only significant near the edges of the holes and is vanishing elsewhere. We can therefore consider the electric field in the middle of the waveguide to be in-plane only. In a previous study Lee et al. measured

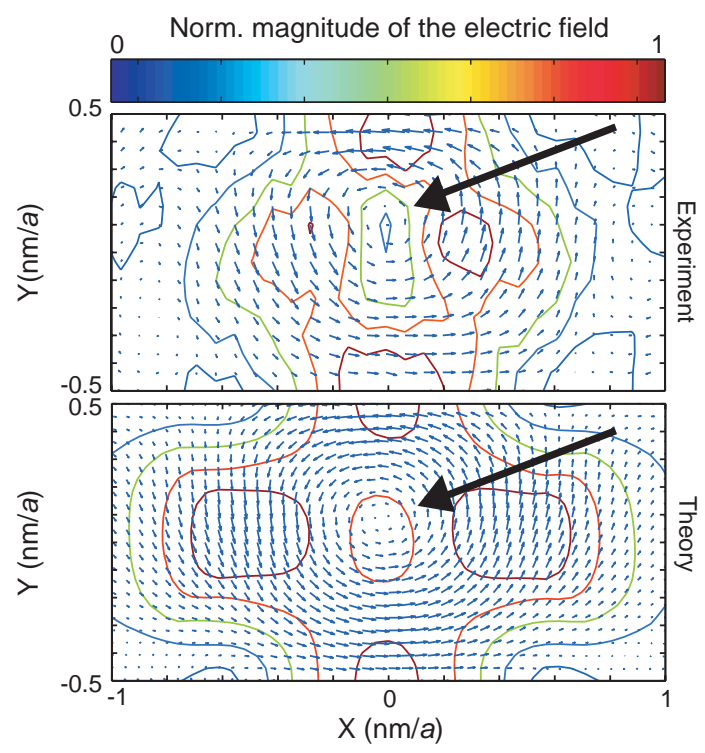

FIG. 3 (color). Experimentally and theoretically obtained instantaneous 2D vector plots of the electric field. The contour plots indicate the normalized magnitude of the electric field. The black arrows highlight a disclination. For both the figures the depicted area is $2 a \times 1 a$ (the center of the waveguide is around $x=0$ ). 
the intensity of the electric field components in the near field [26]. In contrast, we perform phase-sensitive measurements that allow us to determine the actual instantaneous direction of the electric field.

In the instantaneous electric vector field shown in Fig. 3, we find a position where the electric field is at that moment zero (indicated by a black arrow). At this position the polarization state is undetermined. This is a so-called wave disclination. Such disclinations are singularities that move as time progresses. Because around such a singularity the electric field vector describes a complete circle (rotation of $\pm 2 \pi$ ), it has a topological charge of \pm 1 [14]. From the vector arrangement in Fig. 3 we infer that the topological charge equals +1 . Usually, disclinations are studied in far-field transmission investigations in a plane orthogonal to the propagation direction of the light. It has been shown that the arrangement of the vectors surrounding a disclination varies as the time progresses [14]. In contrast, we investigate the polarization state in a plane parallel to the propagation direction and thus obtain insight in the disclination evolution inside the sample that generates it. We observe that the disclination moves as time progresses but the topology around it is in a stable vortex shape. We attribute this to the fact that the disclination moves in the center of the waveguide, where there is a zero crossing in $E_{y}$ [see Fig. 2(f)]. Hence, the electric field must be parallel to the $x$ direction for every point along the center of the waveguide. This condition fixes the topology of the vectors and prevents the deformation of the vortex around the dislocation.

To elucidate the polarization distribution of the light inside the waveguide further, we determine the ratio $\varepsilon=$ $\mp u / v=\tan \{\arcsin [(\sin 2 \psi) \sin \delta] / 2\}$ and the orientation angle $\alpha=\{\arctan [(\tan 2 \psi) \cos \delta]\} / 2$ of the polarization ellipse, where $v$ is the major semiaxis, $u$ is the minor semiaxis [as illustrated in Fig. 4(a)]. The angles $\psi=$ $\arctan \left(\left|E_{y}\right| /\left|E_{x}\right|\right)$ and $\delta=\delta_{y}-\delta_{x}$ characterize the amplitude ratio and the phase difference between the two electric field components, respectively [32]. Thus, to determine $\varepsilon$ and $\alpha$ from the two field components, knowing the phase relation between them is crucial. Figure 4(b) shows $\varepsilon$ retrieved from the measured and calculated vector field distribution of Fig. 3. Negative and positive values correspond to left- and right-handed polarization, respectively. Two areas of opposite handedness are in close proximity (within 2 unit cells, or $860 \mathrm{~nm}$ ). Figure 4(c) depicts the angle $\alpha$ retrieved from the calculated and measured field distribution. Note that, because $\alpha=0$ and $\alpha=\pi$ correspond to the same polarization state, we have chosen a cyclic color map. The phase-sensitive near-field measurements and the calculations show that, in an area of the order of the square wavelength in material $\left[(\lambda / n)^{2}\right]$, the polarization state has an antisymmetric structure.

When the polarization is purely linear $(\varepsilon=0)$, the handedness of the polarization ellipse is undetermined. This occurs along the so-called $L$ lines, which separate (a)

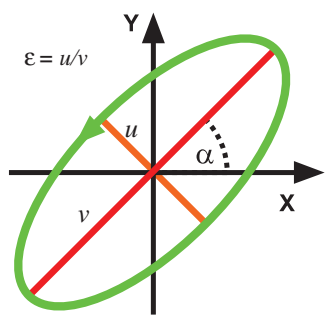

(b)
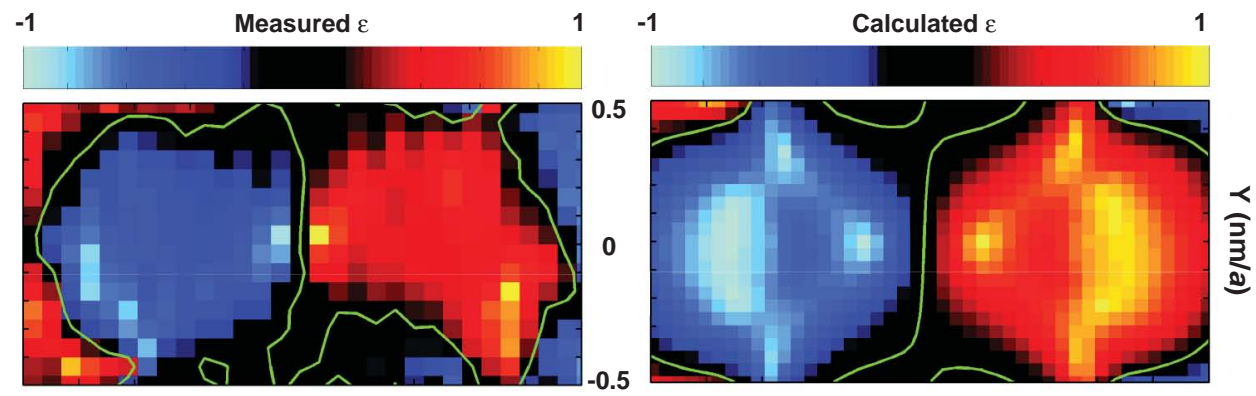

(c)

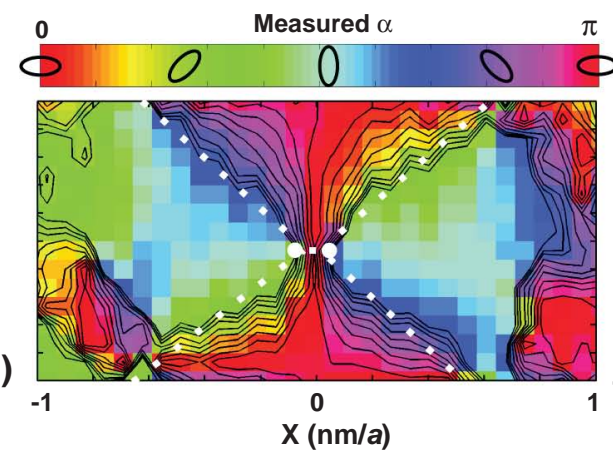

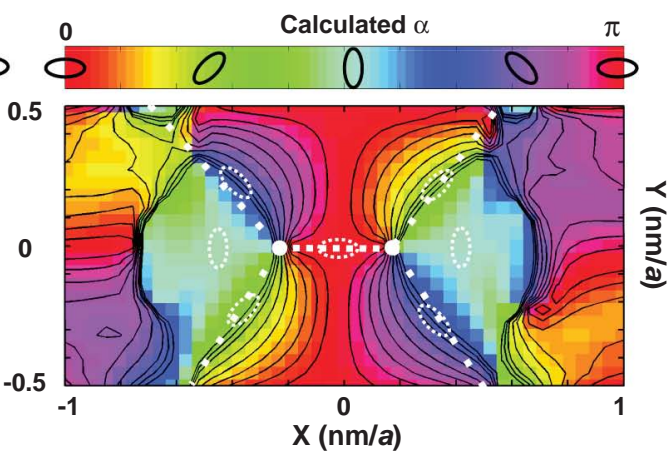

FIG. 4 (color). (a) Representation of the polarization ellipse, where $v$ is the major semiaxis, $u$ is the minor semiaxis of the ellipse, $\varepsilon=\mp u / v$ and $\alpha$ the orientation angle. (b) Experimentally and theoretically obtained $\varepsilon$. Negative and positive values correspond to left- and right-handed polarization, respectively. Lines of linear polarization ( $L$ lines) are shown in green. (c) Experimentally and theoretically obtained $\alpha$. The dotted ellipses indicate the orientation of the polarization and the dashed lines show the threefold symmetry of the system. The white dots indicate the position of $C$ points. The depicted area is $2 a \times 1 a$ (the waveguide is centered around $x=0)$. 
areas of opposite handedness. The above-mentioned polarization disclinations must move along $L$ lines as time progresses [14]. In Fig. 4(b) $L$ lines are indicated by green lines. We find a good match between measurement and calculation. The disclination observed in Fig. 3 lies on the $L$ line in the center of the waveguide for both experiment and theory, as predicted by Nye.

When $\varepsilon= \pm 1$ the polarization is purely circular, which means that the orientation of the angle $\alpha$ is undetermined. Positions where this occurs are referred to as $C$ points. Around such a point the polarization ellipse describes semicircle (rotation of $\pm \pi$ ). Thus, these singularities are characterized by a topological charge of $\pm \frac{1}{2}$, where the sign is determined by the rotation of the direction of the ellipse around the $C$ point [15]. In order to identify $C$ points, we introduce the so-called isogyres, defined as lines of equal $\alpha$. These isogyres are shown in Fig. 4(c) as black lines. The isogyres clearly converge on two points [white dots in Fig. 4(c)] close to the center of the photonic crystal waveguide. Because by definition the isogyres are lines with one unique value of $\alpha$, their intersection is possible only when $\alpha$ is undetermined. Thus, the intersections of the isogyres are $C$ points. The dashed white lines and ellipses in Fig. 4(b) show the symmetry and ellipse orientation around the $C$ points, respectively. Both the $C$ points have topological charge $-\frac{1}{2}$ and a threefold symmetry. This means that this is a so-called star singularity. For further information about the classification of the singularities see Ref. [15]. We observed that the Bloch mode propagating in a photonic crystal waveguide generates in every unit cell two circular polarization singularities with the same topological charge in subwavelength proximity $(200 \mathrm{~nm}$ according to calculation and $50 \mathrm{~nm}$ according to the measurements). In previous publications it has been shown that polarization singularities with the same topological charge repel each other (the so-called topological singularity screening) $[18,20]$. However, as the two $C$ points we investigated have opposite handedness, they are essentially independent and therefore screening does not play a role here.

In conclusion, exploiting the polarization properties of a highly cylindrical symmetric aperture near-field probe, we experimentally visualized the electric vector field of the light propagating through a photonic crystal waveguide. We observed with subwavelength resolution the vectorial topological defects present in the photonic crystal waveguide. We found two star-type circular polarization singularities in subwavelength proximity and a disclination lying on a $L$ line in the center of the waveguide itself. All the measurements are in good agreement with the 3D finite-difference time-domain calculations. The observed dependence of the polarization state can be used for the on chip manipulation of (cold) atoms and may lead to new strategies for quantum information processing with nanophotonic structures.
We wish to thank T. D. Visser helpful discussion. This work is part of the research program of the "Stichting voor Fundamenteel Onderzoek der Materie (FOM)," which is financially supported by the "Nederlandse organisatie voor Wetenschappelijk Onderzoek (NWO)." Support by the NWO (VICI grant) is gratefully acknowledged. This work is also supported by NanoNed, a nanotechnology program of the Dutch Ministry of Economic affairs.

*burresi@amolf.nl

[1] R. A. Gray et al., Nature (London) 392, 75 (1998).

[2] M. V. Berry, Proc. SPIE Int. Soc. Opt. Eng. 4403, 1 (2001).

[3] T. H. R. Skyrme, Proc. R. Soc. A 260, 127 (1961).

[4] J. R. Abo-Shaeer et al., Science 292, 476 (2001).

[5] K. W. Madison et al., Phys. Rev. Lett. 84, 806 (2000).

[6] M. R. Matthews et al., Phys. Rev. Lett. 83, 2498 (1999).

[7] A. A. Abrikosov, Sov. Phys. JETP 5, 1174 (1957).

[8] P. Coullet et al., Phys. Rev. Lett. 62, 1619 (1989).

[9] M. Berry, Nature (London) 403, 21 (2000).

[10] J. F. Nye and M. V. Berry, Proc. R. Soc. A 336, 165 (1974).

[11] H.F. Schouten, T.D. Visser, G. Gbur, D. Lenstra, and H. Blok, Phys. Rev. Lett. 93, 173901 (2004).

[12] N. Shvartsman and I. Freund, Phys. Rev. Lett. 72, 1008 (1994).

[13] S. Zhang and A.Z. Genack, Phys. Rev. Lett. 99, 203901 (2007).

[14] J. F. Nye, Proc. R. Soc. A 387, 105 (1983).

[15] J. F. Nye, Proc. R. Soc. A 389, 279 (1983).

[16] J. V. Hajnal, Proc. R. Soc. A 414, 447 (1987); J. V. Hajnal, Proc. R. Soc. A 430, 413 (1990).

[17] R. W. Schoonover and T. D. Visser, Opt. Express 14, 5733 (2006).

[18] F. Flossmann et al., Phys. Rev. Lett. 95, 253901 (2005).

[19] M. V. Berry, M. R. Dennis, and R. L. Lee, New J. Phys. 6, 162 (2004).

[20] R. I. Egorov, M. S. Soskin, D. A. Kessler, and I. Freund, Phys. Rev. Lett. 100, 103901 (2008).

[21] F. Flossmann, K. O‘Holleran, M. R. Dennis, and M. J. Padgett, Phys. Rev. Lett. 100, 203902 (2008).

[22] D. Rychtarik, B. Engeser, H.-C. Nägerl, and R. Grimm, Phys. Rev. Lett. 92, 173003 (2004).

[23] W. Hansel, P. Hommelhoff, T. W. Hansch, and J. Reichel, Nature (London) 413, 498 (2001).

[24] J. Bravo-Abad, M. Ibanescu, J. D. Joannopoulos, and M. Soljacic, Phys. Rev. A 74, 053619 (2006).

[25] I. Fushman et al., Science 320, 769 (2008).

[26] K. G. Lee et al., Nat. Photon. 1, 53 (2007); H. Gersen et al., ibid. 1, 242 (2007); K. G. Lee et al., ibid. 1, 243 (2007).

[27] Z. H. Kim and S. R. Leone, Opt. Express 16, 1733 (2008).

[28] H. Fischer et al., J. Microsc. 230, 27 (2008).

[29] J. A. Veerman et al., Appl. Phys. Lett. 72, 3115 (1998).

[30] M. Sandtke et al., Rev. Sci. Instrum. 79, 013704 (2008).

[31] R. J. P. Engelen et al., Nature Phys. 3, 401 (2007).

[32] M. Born and E. Wolf, Principle of Optics (Cambridge Univ. Press, Cambridge, 1999), 7th ed. 